(C) 2016 IEEE. Personal use of this material is permitted. Permission from IEEE must be obtained for all other uses, in any current or future media, including reprinting/republishing this material for advertising or promotional purposes, creating new collective works, for resale or redistribution to servers or lists, or reuse of any copyrighted component of this work in other works. 


\section{Guest Editorial: Sensor Systems for a Changing Ocean}

Oceans regulate the Earth's climate and are integral to all known sources of life. Ocean processes are of biological, geological, chemical or physical nature, occurring at micro- to kilometer scales, from less than seconds to centuries, turning the understanding and the sustainable management of the ocean into a multi-scale and multi-disciplinary effort. Collection of in-situ observation of a volume that covers over $70 \%$ of the planet is also inherently challenging and remains generally difficult and costly in time and resources, with so far a rather unsatisfactory result, in particular with respect to space-time resolution. Over the past decade, there has been a steady crescendo of interest to support the development of a truly integrated and sustainably funded Ocean Observing System. This will be achieved with more long-term measurements of key parameters but is impaired by the costs and lack of reliability of ocean sensors in general.

These topics are well represented in this special issue of Sensor Systems for a Changing Ocean: underwater sensors, the next generation (SSCO), and the papers presenting them give some very fresh looks into these themes. The contributions span new, integrated sensors that can be implemented on a variety of fixed and mobile platforms and have multiple functionalities including measurements of key parameters useful to a number of objectives, ranging from more precise monitoring and modeling of the marine environment to an improved assessment of fish stocks.

In Long-term in situ survey of reactive iron concentrations at the EMSO-Azores observatory, authored by Laës-Huon, Cathalot, Legrand, Tanguy and Sarradin, a study of the temporal dynamics of iron concentrations and temperature at an Atlantic Ocean deep sea vent was performed. They utilized the innovative CHEMINI in situ analyzer at the Lucky Strike hydrothermal field investigating its analytical reliability and mechanical robustness over a period of 6 months.

In In situ observations of biological and environmental parameters by means of optics Development of next ocean sensors with special focus on an integrating cavity approach, Wollschläger, Voß, Zielinski and Petersen present two optical sensor developments of the NeXOS project. One development is for continuous hyperspectral absorption measurement based on integrating cavity approach. The second development is a matrix-fluorescence sensor with flexible wavelength configuration for the detection and characterization of dissolved substances, such as fluorescent dissolved organic matter and PAHs.

In TS-MUWSN: Time Synchronization for Mobile Underwater Sensor Networks, Pallares, Bouvet and del Rio Fernandez propose a novel method for synchronization, and Doppler scale estimation in underwater acoustic communications. The technique uses DASync time synchronization algorithm and to compensate Doppler distortion three different frequency shift estimators have been evaluated with the synchronization protocol.

In Development of wireless and passive corrosion sensors for material degradation monitoring in coastal zones and immersed environment, Khalifeh, Yasri, Lescop, Gallee, Diler, Theirry and Rioual present a novel wireless and passive corrosion sensors inspired from the chipless Radiofrequency Identification (RFID) technology. The proposed sensor which should be able to be deployed in 
immersed, tidal and splash zones, can be considered as a reliable and versatile tool for example for offshore facilities monitoring.

We believe that these papers highlight some of best recent ideas in the field of monitoring the marine environment through the implementation of innovations, such as multiplatform integration better sensor stability management and improved sensor as well as data interoperability that provide greater reliability. , The collection of papers provides an excellent starting point for researchers who want to contribute to this field.

Daniel Mihai Toma, Guest Editor

SARTI research group [Electronics Dept] of Technical University of Catalunya (UPC)

Oliver Zielinski, Guest Editor

Institute for Chemistry and Biology of the Marine Environment, University of Oldenburg, Germany 